\title{
Deterministic-based power grid planning enhancement using system well-being analysis
}

\author{
Wijarn WANGDEE ${ }^{1}$
}

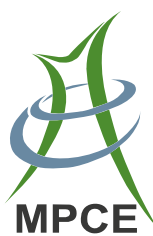

\begin{abstract}
This paper presents a comprehensive approach to enhance the traditionally deterministic-based power grid planning using system well-being analysis concept. The objective is to identify and characterize the existing system reliability concerns inherited from the adopted deterministic criteria, so that power utilities can accordingly adjust their reliability criteria to cope with real-life system uncertainties and hence to enhance the overall system reliability. A determination of transmission capacity reserve derived from incorporating deterministic criteria into a probabilistic framework has been introduced in the paper. An application of the proposed methodology for justifying a transmission addition project to accommodate the system load growth is illustrated by using an actual island power grid. Both technical and economic aspects, greatly concerned in practice, have been taken into consideration in the project justification.
\end{abstract}

Keywords Deterministic criteria, Economic analysis, Reliability, Security, Transmission capacity reserve

CrossCheck date: 14 December 2017

Received: 30 December 2016/Accepted: 14 December 2017/ Published online: 23 February 2018

(C) The Author(s) 2018. This article is an open access publication

Wijarn WANGDEE

wijarn@ieee.org

1 The Sirindhorn International Thai-German Graduate School of Engineering (TGGS), King Mongkut's University of Technology North Bangkok (KMUTNB), Bangkok 10800, Thailand

\section{Introduction}

Power utilities around the globe have been using deterministic criteria in bulk power grid planning and operation for decades. This is because the traditional deterministic criteria offer several favorable aspects such as uncomplicated concept, simple implementation, and solid judgment. When considering bulk power system reliability, most power utilities utilize the deterministic rule designated as "N-1 security criterion", which implies that the system should be able to withstand any major single component outage without violating the operating criteria and causing the loss of load.

In general, the traditional deterministic approach specifies operating system conditions with some assumptions and then analyzing whether or not the loss of a single circuit or generator will result in a violation of the operating criteria. Deterministic measures only signify that either the system meets or does not meet the given criteria, but do not make any guiding information available on the likelihood of the system to meet the criteria. Consequently, this practice exhibits the drawback as all the resulting limits are inflexible outcomes, in which there is no mechanism for adjusting the deterministic measures as a function of the probability or consequence of the contingency occurring [1].

Basically, deterministic reliability criteria are used to limit the stress level of a system without creating unacceptable risk. Reliability criteria essentially determine the balance between reliability and the allowable system utilization in system planning and operation. Power system restructuring compounded with high penetration of intermittent renewable energy sources creates an increased transmission utilization resulting in an increase in the system stress level. The design, operation and application 
of deterministic criteria to properly balance the utilization and reliability of power systems will become increasingly challenging in the changing system environment. A transmission transfer limit determined solely by the $N-1$ security criterion may no longer be sufficient under new power system paradigms [2].

The essential weakness of the $N-1$ criterion is basically due to the fact that it does not incorporate the stochastic nature of power system behavior and therefore does not provide an actual system reliability assessment [3]. For this reason, the deterministic $N-1$ criterion may not be able to ensure that the system security will remain sufficient for future systems involving greater uncertainties. The deterministic $N-1$ criterion is, however, definitely easier for engineers, managers and regulators to appreciate than quantitative indices obtained using probabilistic approaches. This conflict can be resolved by incorporating the deterministic $N-1$ criterion into a probabilistic framework. This concept is designated as system well-being analysis $[4,5]$, which provides a mechanism to augment the traditional deterministic criteria using probabilistic analysis process. There are also alternative ways to take both deterministic and probabilistic criteria into consideration. References [6-8] present the transmission planning process by initially investigating all transmission reinforcement options (alternatives) that meet the deterministic criteria, and those reinforcement alternatives are then ranked based on their benefits derived from the probabilistic method. With this approach, the final selected reinforcement alternative can comply with both deterministic and probabilistic criteria. Risk-based or probabilistic risk assessment (PRA) methodology presented by EPRI [9-11] is another practical hybrid approach that combines a probabilistic measure of the likelihood of undesirable events with a measure of the consequence of the events (that is, the impact) into a single reliability index. In the PRA method, deterministic contingency events are weighted by their occurrence probabilities and then multiplied with their consequences to create the risk index. As a result, the traditional deterministic criteria have been inherently loosen from the planning process. This is different from the well-being analysis concept where the deterministic criteria are still explicitly taken into consideration. The purpose of utilizing system well-being analysis in the study is not to directly replace deterministic criteria with probabilistic reliability considerations, but the intent is to identify and characterize the actual (existing) system reliability concerns inherited from the specified deterministic reliability criteria so that power utilities can adjust and expand these criteria to meet the new stochastic realities.

This paper integrates the concept of the locational transmission capacity reserve described in [12] into a practical transmission planning process considering both reliability and economic considerations. The locational transmission capacity reserve is defined as the additional transmission capacity required to deliver the electrical power from remote generation to supply the load demand in a generation deficit area (load dominant area), i.e. through the inter-tie lines. In some generation deficit areas that rely heavily on inter-tie lines to access remote generation from neighboring areas, a stringent deterministic security rule such as the $N-1-G$ criterion in which a loss of single transmission component together with a loss of critical local generating unit is considered [12, 13]. Another example of $N-1-G$ application is when considering system security under the generating unit maintenance scheduling along with the $N-1$ contingency condition of the transmission system [14]. The utilization of the $N-1-G$ security criterion in transmission planning, on one hand, could improve the system reliability. On the other hand, the resulting reliability level associated with this criterion may not be consistent and may involve significant cost if the size of the critical local generating unit in the studied area is quite large leading to a substantial amount of transmission capacity being reserved under the specified security criterion [12]. However, the method presented in [12] only aims at determining the additional transmission capacity required as the reserve for accessing the remote generation to secure the system load based solely on the reliability standpoint while ignoring other relevant technical and economic considerations.

In contrast, this paper extends the concept proposed in [12] by applying it to a more comprehensive transmission planning process typically employed in practice, where other relevant technical and economic analyses must also be considered. Consequently, the contribution of this paper is aimed at demonstrating that the method presented in [12] is not only technically sound when incorporating the deterministic criteria into the probabilistic framework, but it is also economic justified when applying it comprehensively to the practical transmission planning process.

In this paper, the study process starts with translating the deterministic criteria into probabilistic measures using system well-being analysis. Once the probabilistic index driven by the given deterministic criteria has been established, this index can be used as a benchmark value to determine the transmission capacity reserve requirement. The proposed methodology has been applied to a real-life scenario where the system load continues to grow, and thus the system reinforcement in a form of a new transmission circuit addition is required to maintain the overall system reliability. Advancing the new transmission addition project in order to accommodate the proposed transmission capacity reserve can offer several benefits such as reducing energy loss, reducing reliability must run (RMR) generation cost, and reducing customer damage cost due to power 
supply failures. However, the advancement of the project will incur an earlier investment cost than the original plan governed by the traditional deterministic criterion. Therefore, the economic analysis to appreciate the present value (PV) of the advanced project must be considered to determine whether or not the project advancement can solidly have an economic justification.

This paper is organized as follows. Section 2 explains the system well-being analysis concept. Section 3 presents the study system and conditions used in the paper. The capacity reserve determination process is then described in Section 4. Section 5 demonstrates the real-life transmission planning application for justifying the advancement of the new transmission addition project in order to facilitate the proposed transmission capacity reserve requirement. Section 6 presents the conclusions.

\section{System well-being analysis}

A bulk power system, alternatively known as composite generation and transmission system, can be divided into the two distinct aspects of adequacy and security [15]. System adequacy relates to the existence of sufficient facilities within the system to satisfy the customer load demand whereas system security relates to the ability of the system to respond to disturbances arising within that system, i.e. $N-1$ contingency events. The bulk power system wellbeing analysis framework [4, 16-18] is the simplified version of the traditional operating states classified by the degree to which adequacy and security constraints are satisfied [19-21].

Deterministic $N-1$ security criterion has been used by most power utilities as a means to maintain bulk power system reliability. Hence, the system well-being concept incorporates the simplified operating states, associated with the accepted deterministic $N-1$ criterion, into a probabilistic framework. The bulk power system well-being structure consists of the three states of healthy, marginal, and at-risk, as shown in Fig. 1 [4, 16-18] where both healthy and marginal states are considered as the success state (i.e. no loss of load) while the at-risk state is a failure state (i.e. having loss of load). In the healthy state, all

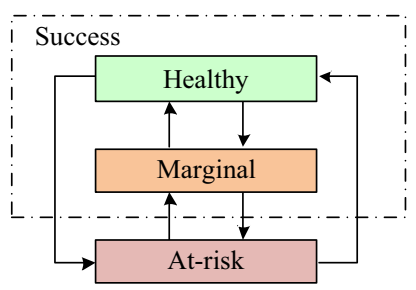

Fig. 1 System well-being framework equipment and operating constraints are within limits, and there is sufficient margin to serve the total load demand, even with the loss of any element ( $N-1$ contingency), i.e. generator or transmission line. In the marginal state, the system is still operating within limits, but there is no longer sufficient margin to satisfy the acceptable deterministic $N-1$ criterion. In the at-risk state, equipment or system constraints are violated, and load is curtailed. Thus, the system well-being analysis presents a combined deterministic and probabilistic framework that offers a quantitative interpretation of the degree of system security (healthy state) and insecurity (marginal state) in a bulk electric power system in addition to the traditional risk measures. It is worth noting that the traditional adequacy assessment refers to the reliability evaluation of the at-risk states only (i.e. encountering loss of load), without concerning the insecure load conditions (but no loss of load) in the marginal states.

When system states that used to reside in the healthy state move or transition to the marginal state, the system becomes stressed and the probability of the marginal state increases. Such system conditions become more difficult for system operators to manage when the system is under $N-1$ situations. In contrast, if the system is planned or designed to have a higher healthy state probability, the system will be more secure and robust from both planning and operation perspectives. Knowledge of system state movements, especially when they move from healthy to marginal, is very crucial and these movements cannot be captured using traditional probabilistic adequacy assessment until they actually move into the at-risk region where they have severe impacts due to the loss of load. The movements from secure states (i.e. healthy) to insecure states (i.e. marginal) are not easily identified if only at-risk states are considered since the at-risk state probabilities do not change to any significant degree [4].

Therefore, system well-being analysis is extremely useful for bridging the gap between the deterministic and probabilistic approaches by quantifying the likelihood of encountering marginal system states (insecure states, but no loss-of-load) as well as that of encountering system atrisk states (loss-of-load states). A detailed procedure for bulk power system well-being analysis can be found in [18]. The quantitative measures of system well-being are in forms of the probabilities, frequencies and durations of the healthy, marginal and at-risk states. In this paper, only the probabilities of the three states are presented, and the healthy state probability is proposed as the key index to be utilized in the transmission planning application. 


\section{Study system and conditions}

The study system used in this paper is an actual bulk power grid serving the load demand on an island in Canada as shown by the simplified single line diagram in Fig. 2 . Forced outage rates (FORs) of all generating units and transmission circuits depicted in Fig. 2 were obtained from historical outage statistics and presented in "Appendix A". In Fig. 2, the continuous lines (black) indicate existing transmission circuits whereas the dashed line (red) presents the new transmission circuit to be added in the future as identified from the long-term transmission planning process. From a bulk transmission supply capability standpoint, the present island system is connected to the mainland through two $500 \mathrm{kV}$ AC submarine cable circuits with ratings of $1200 \mathrm{MW}$ each and one $230 \mathrm{kV}$ AC submarine cable circuit with a rating of $600 \mathrm{MW}$. Power flow on the $230 \mathrm{kV}$ circuit is controlled by a phase shifting transformer to prevent thermal overload under a contingency event when one of the $500 \mathrm{kV}$ circuits is forced out of service, i.e. the worst single contingency for the bulk transmission supply to the island. From local generation capacity, the total local installed generating capacity on the island is $692 \mathrm{MW}$. The present total load demand on the island is about $2225 \mathrm{MW}$. Hence, the island system is considered as the generation deficit area (i.e. load dominant area) where the bulk power flows from the mainland towards the island. The amount of appropriate transmission transfer capability through the bulk supply system from the mainland to the island is, therefore, of considerable interest.

From transmission planning perspective, power flow on the $230 \mathrm{kV}$ circuit is fully controlled by the phase shifting transformer and is fixed at $600 \mathrm{MW}$ for planning study

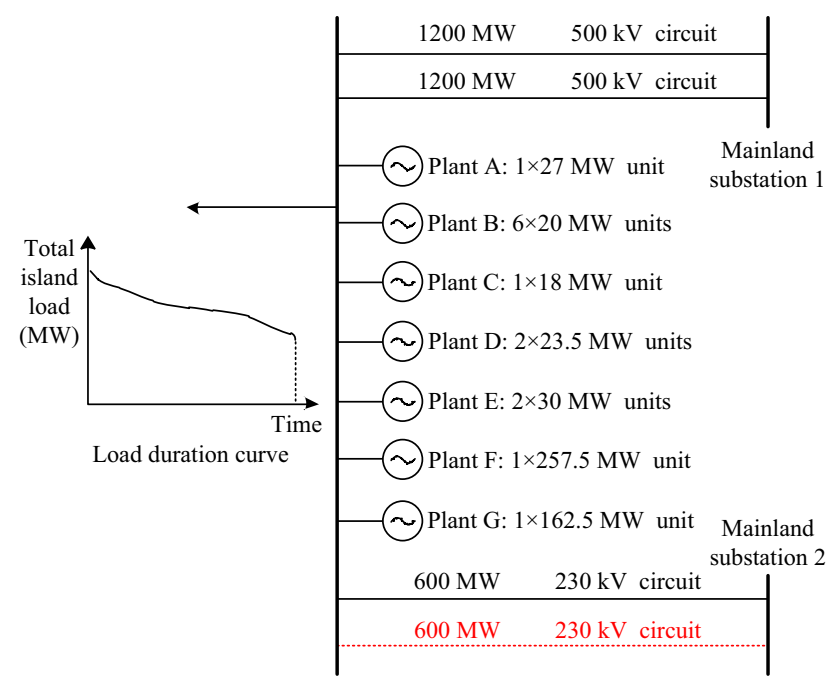

Fig. 2 A simplified power supply system to the island purposes. The existing firm transfer capability of the bulk transmission supply system based on the deterministic $N-1$ criterion is constrained by a loss of one of the $500 \mathrm{kV}$ circuits (i.e. the worst $N-1$ contingency), and is equal to $1800 \mathrm{MW}$ (i.e. $1200 \mathrm{MW}$ of the $500 \mathrm{kV}$ circuit plus $600 \mathrm{MW}$ of the $230 \mathrm{kV}$ circuit). The island supply capability based on the deterministic $N-1$ criterion is the sum of the firm transfer capability and the local generation, and is $2492 \mathrm{MW}$ (i.e. $1800+692$ ). The present island load is at $2225 \mathrm{MW}$, and therefore the island supply system still has a transfer capability margin of 267 MW (i.e. 2492-2225) for accommodating a future load growth.

\section{Combined deterministic and probabilistic concepts for capacity reserve determination}

A state sampling Monte Carlo simulation [22] approach was used to evaluate the system well-being indices. This simulation approach was used in the study because the method is more flexible and can be easily extended to incorporate other relevant uncertainty factors. The load duration curve shown in Fig. 2 was obtained from the historical annual load profile of the island system. The coefficient of variation (i.e. a stopping criterion) based on the expected energy not supplied (EENS) index was set at $2 \%$. The study procedure firstly describes practical deterministic criteria used by power utilities, followed by a translation of the deterministic criteria into probabilistically quantifiable indices, and a determination of transmission capacity reserve.

\subsection{Deterministic transmission planning criteria}

In bulk power system planning, the deterministic $N-1$ criterion has been used by most power utilities for many years to assess the transmission transfer capability (TC) requirement. Conceptually, the TC indicates the maximum transfer capability of the transmission system under the $N-1$ condition, generally known as the firm transfer capability. For the transmission supply to the island system, the existing TC is $1800 \mathrm{MW}$, which is the sum of one of the $500 \mathrm{kV}$ circuit capacity and the $230 \mathrm{kV}$ circuit capacity (i.e. $1200+600$ ).

From traditional system planning practice, a maximum transfer demand (TD) or a committed use of the future load and resource scenario must be acknowledged in order to determine whether or not system reinforcements will be required. The system reinforcement is required to relieve the future congestion whenever the TD exceeds the TC based on the $N-1$ criterion [23]. TD on the transmission path in question, which is a specified set of transmission circuits delivering power from one area to another area, is 
equal to the total receiving-end load less the total receiving-end generation. Therefore, the present TD for the island system is $1528 \mathrm{MW}$ (i.e. 2225-697). This indicates that the existing island system still has the TD less than the $\mathrm{TC}$, and therefore the transmission system reinforcement is not yet required for the present load demand condition based on the traditional deterministic criteria.

Based on the above explanation, the three deterministic transmission planning rules can be summarized as follows:

1) TC of the transmission system is determined by considering the worst $N-1$ contingency condition (i.e. a $500 \mathrm{kV}$ circuit outage in this case).

2) Transfer demand $=$ total local load - total local generation.

3) System reinforcement is required when the TD exceeds the TC in order to eliminate the transmission system congestion.

\subsection{Translation of deterministic criteria into probabilistic criteria}

The three deterministic rules described in Section 4.1 are translated into a probabilistic index using the system well-being analysis concept described in Section 2. In order to quantify the degree of system reliability inherited from the deterministic rules, the total island load was scaled up to $2492 \mathrm{MW}$ to represent the maximum load demand on the island that can be supplied by the $N-1$ firm TC from the mainland along with the supply from the local generation on the island (i.e. $1800+692=2492 \mathrm{MW}$ ). Under this condition, the TD is equal to TC and therefore any further load increase will violate the deterministic criteria according to the third deterministic rule previously described.

The second deterministic rule $(\mathrm{TD}=$ total local load - total local generation) implies that all the local generation is always fully available. In other words, all the local generating units are assumed to be $100 \%$ reliable under this deterministic rule. Therefore, the FORs of all the local generating units on the island are set to zero based on this deterministic criterion translation. In some utility practice [24], transmission capacity requirements are determined based on the assumption that all generating capacities are dependable or all available (i.e. ignoring generating unit forced outages). Such a planning assumption, consequently, was tested and verified in the paper to encourage power utilities regarding what needs to be improved in the traditional planning process.

In the first deterministic rule (TC based on the $N-1$ condition), the deterministic $N-1$ criterion is incorporated in the system well-being analysis framework. In the analysis, the unavailabilities assigned to the 230 and $500 \mathrm{kV}$ transmission circuits are based on historical outage data. The system well-being indices shown in Table 1 are a direct translation of the three deterministic rules described in Section 4.1. These deterministic-based system well-being indices are solely driven by the failure probabilities of the transmission circuits, whereas the failure probabilities of all the local generating units are not considered (i.e. all generators are assigned a zero FOR).

The system well-being indices shown in Table 1 are considered as the benchmark indices directly related to the utilization of the traditional deterministic approach, which are then used to compare against the results obtained by taking the local generating unit FOR into consideration as presented in the next subsection. The probability of healthy state $\left(P_{\mathrm{H}}\right)$ shown in Table 1 is designated as the "deterministic-based $P_{\mathrm{H}}$ " that is used as a key index to determine the transmission capacity reserve in this paper.

\subsection{Transmission capacity reserve determination}

Considering all the local generating units to be always fully available (i.e. zero FOR) is obviously highly optimistic because the unavailability of generating units are generally much higher by an order of magnitude than the unavailability of transmission circuits. Table 2 presents the system well-being indices for the same system conditions as those demonstrated in Table 1 except that the actual FOR (i.e. non-zero FOR) of all local generators have been incorporated. By comparing the deterministic-based $P_{\mathrm{H}}$ shown in Table 1 against the resulting $P_{\mathrm{H}}$ in Table 2, it can be clearly observed that the real-life system when considering all possible generation and transmission unavailabilities (Table 2) is actually not as secure (healthy) as that defined by the benchmark value, i.e. the deterministicbased $P_{\mathrm{H}}$ given in Table 1 .

In real-life, all generators can fail resulting in a lower $P_{\mathrm{H}}$ as shown in Table 2 when compared to the deterministicbased $P_{\mathrm{H}}$ shown in Table 1 . This lower $P_{\mathrm{H}}$ from Table 2 is, however, more realistic as all generators with non-zero FOR had been incorporated. To retain the system security at the similar level to the deterministic-based $P_{\mathrm{H}}$, the transmission capacity reserve must be reconsidered in order to offset the less dependable generators due to the non-zero FOR consideration. The amount of the

Table 1 Deterministic-based system well-being indices of island system when $\mathrm{TC}=\mathrm{TD}$, considering all generators with zero FOR

\begin{tabular}{ll}
\hline System well-being indices & Load at 2492 MW \\
\hline Probability of healthy state $\left(P_{\mathrm{H}}\right)$ & 0.99188 \\
Probability of marginal state $\left(P_{\mathrm{M}}\right)$ & 0.00810 \\
Probability of at-risk state $\left(P_{\mathrm{R}}\right)$ & 0.00002 \\
\hline
\end{tabular}


Table 2 System well-being indices of island system when TC $=$ TD, considering all generators with non-zero FOR

\begin{tabular}{ll}
\hline System well-being indices & Load at $2492 \mathrm{MW}$ \\
\hline Probability of healthy state $\left(P_{\mathrm{H}}\right)$ & 0.98832 \\
Probability of marginal state $\left(P_{\mathrm{M}}\right)$ & 0.01164 \\
Probability of at-risk state $\left(P_{\mathrm{R}}\right)$ & 0.00004 \\
\hline
\end{tabular}

transmission capacity reserve can be reasonably determined by varying the island peak load levels until the resulting $P_{\mathrm{H}}$ becomes the same as the deterministic-based $P_{\mathrm{H}}$. The difference between the given island peak load and the maximum island load (i.e. $2492 \mathrm{MW}$ ) indicates the appropriate transmission capacity reserve that should be provided. The system well-being indices associated with generators having non-zero FOR were examined at various island peak load levels as presented in Table 3 and also depicted in Fig. 3. The concept for determining the transmission capacity reserve is correspondingly illustrated in Fig. 3.

In Fig. 3, the straight line indicates the deterministicbased $P_{\mathrm{H}}$ of 0.99188 translated from the deterministic rules, which should be held constant at any load conditions as a specified deterministic security level.

The probabilities of the healthy, marginal and at-risk states at various load levels are presented by green, yellow and red color, respectively. The present island peak load is at $2225 \mathrm{MW}$ indicating the TD still less than the TC, and therefore the $P_{\mathrm{H}}$ at $2225 \mathrm{MW}$ peak load is higher than the deterministic-based $P_{\mathrm{H}}$ indicating a more secure system condition. At each load level, the three probabilities are added up to 1.0 (i.e. $P_{\mathrm{H}}+P_{\mathrm{M}}+P_{\mathrm{R}}=1$ ). $P_{\mathrm{R}}$ is typically much smaller by a few orders of magnitude than $P_{\mathrm{M}}$, and therefore $P_{\mathrm{R}}$ is not obviously noticed from Fig. 3. In

Table 3 System well-being indices at various peak load levels, considering all generators with non-zero FOR

\begin{tabular}{llll}
\hline Island peak load $(\mathrm{MW})$ & $P_{\mathrm{H}}$ & $P_{\mathrm{M}}$ & $P_{\mathrm{R}}$ \\
\hline 2225 & 0.99516 & 0.00483 & 0.00001 \\
2250 & 0.99457 & 0.00542 & 0.00001 \\
2275 & 0.99439 & 0.00560 & 0.00001 \\
2300 & 0.99369 & 0.00629 & 0.00002 \\
2325 & 0.99342 & 0.00656 & 0.00002 \\
2350 & 0.99258 & 0.00740 & 0.00002 \\
2375 & 0.99155 & 0.00842 & 0.00002 \\
2400 & 0.99138 & 0.00860 & 0.00003 \\
2425 & 0.99073 & 0.00924 & 0.00003 \\
2450 & 0.98985 & 0.01011 & 0.00003 \\
2475 & 0.98930 & 0.01067 & 0.00003 \\
2492 & 0.98832 & 0.01164 & 0.00004 \\
\hline
\end{tabular}

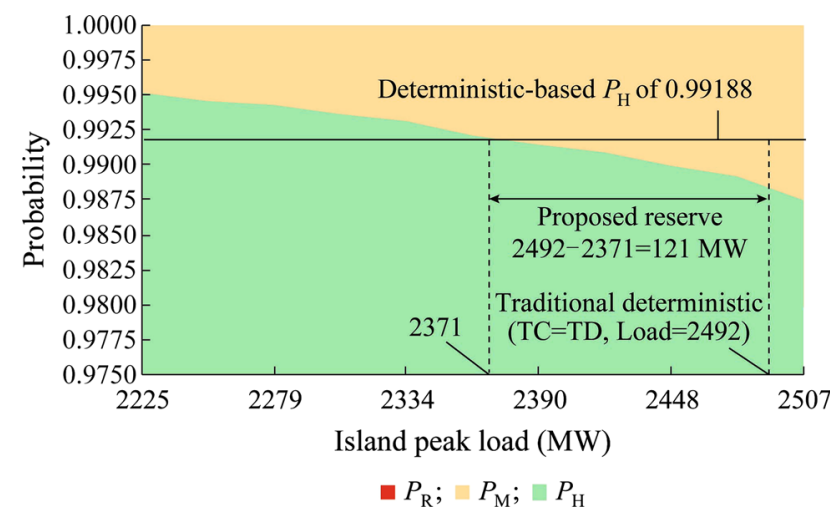

Fig. 3 Determination of transmission capacity reserve for island system

addition, when the system load increases, $P_{\mathrm{R}}$ does not change at a significant degree compared to dramatic changes of $P_{\mathrm{H}}$ and $P_{\mathrm{M}}$ as clearly observed in Fig. 3 .

When the island peak load is at $2371 \mathrm{MW}$, the resulting $P_{\mathrm{H}}$ is equal to the deterministic-based $P_{\mathrm{H}}$ of 0.99188 , which indicates that the transmission capacity reserve should be computed at this load level where the resulting $P_{\mathrm{H}}$ is held at the same value as the deterministic-based $P_{\mathrm{H}}$. Therefore, the proposed transmission capacity reserve is $121 \mathrm{MW}$ as defined by the difference between the maximum island load (i.e. $2492 \mathrm{MW}$ ) and the load level at which the resulting $P_{\mathrm{H}}$ is equal to the deterministic-based $P_{\mathrm{H}}$ (i.e. $2371 \mathrm{MW}$ ) illustrated in Fig. 3.

\section{Transmission planning application}

The defined deterministic planning criteria together with the proposed transmission capacity reserve derived from the previous section are taken into consideration for the real-life transmission planning application illustrated in this section. Practical planning process normally utilized by power utilities is conducted in this paper in order to demonstrate a comprehensive transmission planning using a combined deterministic and probabilistic framework. The 15 -year long range plan with the annual load growth rate of $1.5 \%$ is considered in this study.

\subsection{Transmission planning alternatives}

There are two transmission planning alternatives considered in this study. The first alternative is based on the traditional deterministic criteria in which a new $230 \mathrm{kV}$ transmission circuit is added when TD exceeds TC (i.e. the island peak load exceeds $2492 \mathrm{MW}$ ). This alternative is designated as "Alternative 1: traditional plan with no reserve". The second alternative is based on incorporating the proposed transmission capacity reserve into the system 
planning consideration. This means that the new $230 \mathrm{kV}$ transmission circuit shall be required when the island load reaches $2371 \mathrm{MW}$, which implies to an advancement of the new $230 \mathrm{kV}$ transmission project by 4 years (according to the $1.5 \%$ annual load growth rate) earlier than the traditional plan. This second alternative is designated as "Alternative 2: advanced plan with reserve".

Figure 4 presents the system well-being indices associated with an increase in the island load associated with an addition of the new $230 \mathrm{kV}$ transmission project when the island load exceeds 2492 MW according to the traditional plan without considering the reserve (Alternative 1). A significant improvement on $P_{\mathrm{H}}$ can be observed once the new $230 \mathrm{kV}$ transmission circuit is added, indicating the island system becomes more secure with the new circuit addition.

Figure 5 presents the system well-being indices associated with an increase in the island load associated with an addition of the new $230 \mathrm{kV}$ transmission project according to the advanced plan considering the reserve (Alternative 2) when the island load exceeds $2371 \mathrm{MW}$ causing the $P_{\mathrm{H}}$ to drop below the deterministic-based $P_{\mathrm{H}}$.

The shaded area within the dashed line implying to the benefit gained from the 4-year advancement of the new $230 \mathrm{kV}$ transmission project, in which the insecure probability $\left(P_{\mathrm{M}}\right)$ has been replaced by the secure probability $\left(P_{\mathrm{H}}\right)$ leading to a very secure and robust system when the transmission capacity reserve is provided.

\subsection{Project benefit quantification}

Benefits from adding the new $230 \mathrm{kV}$ transmission project can be considered in both forms of hard and soft benefits. Hard benefits are those components that evidently link to direct expenditure to the power utility. These hard benefits are energy loss (transmission loss) reduction due to transmission system reinforcement, and RMR generation cost reduction resulted from system security enhancement. In contrast, the soft benefits are those financial components

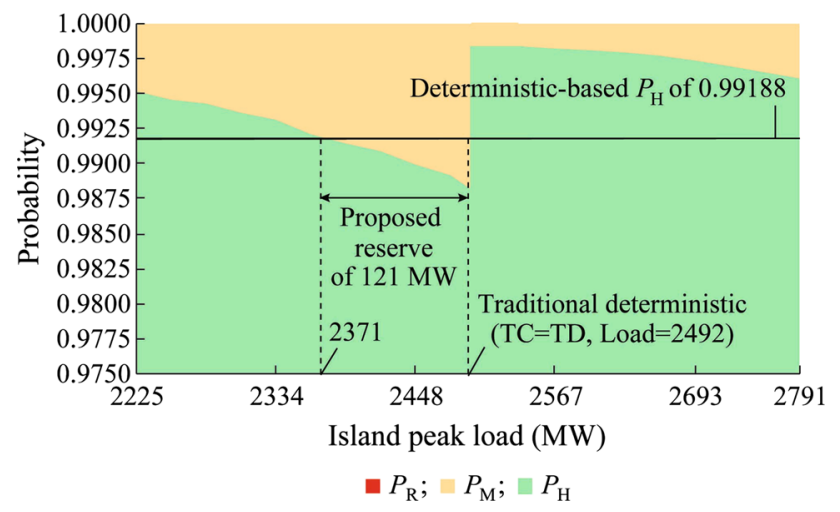

Fig. 4 System well-being indices associated with Alternative 1

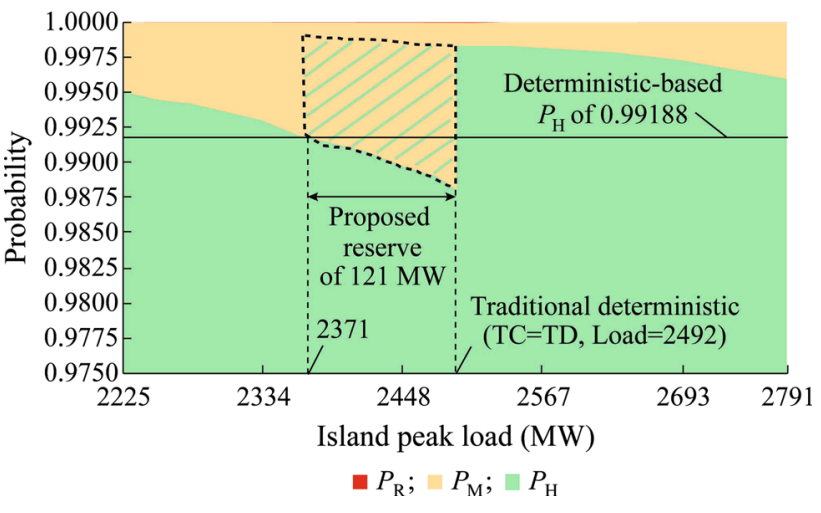

Fig. 5 System well-being indices associated with Alternative 2

incur to customer loads (end users). The soft benefit investigated in this study is the customer damage cost reduction due to the power supply reliability improvement.

\subsubsection{Energy loss reduction}

An addition of the $230 \mathrm{kV}$ transmission circuit will result in reduction in energy loss. Therefore, the advancement of the $230 \mathrm{kV}$ transmission addition project by 4 years can offer an earlier energy loss saving to the power utility. The annual energy loss was calculated based on the following considerations.

1) The detailed 230 and $132 \mathrm{kV}$ regional transmission networks on the island, which is not shown in Fig. 2, were considered in the power flow study and energy loss evaluation in order to reasonably obtain the realistic results.

2) Historical hourly load duration curve was considered $(8760 \mathrm{~h})$.

3) The unit cost of energy is assumed to be $80 \$ / \mathrm{MWh}$.

4) The 15-year long range plan has been considered by starting from the year when the deterministic-based $P_{\mathrm{H}}$ is violated. The violating year (i.e. a year where the peak load exceeds $2371 \mathrm{MW}$ ) is considered as Year 1 in the 15-year long-range plan in which the advanced schedule for the transmission addition project is considered in this year, whereas the normal schedule solely followed the traditional deterministic criteria is considered to add the new transmission circuit in Year 5 (i.e. 4 years later) when the peak load exceeds 2492 MW (i.e. TD > TC).

\subsubsection{RMR cost reduction}

A requirement of RMR generation is necessary in some circumstances to ensure that the electric system is operated within reliability criteria for meeting the load and providing security support in constrained areas. Typically, power 
utilities call for RMR requirements in all situations when generation is impacted due to the transmission system constraints [25].

In many utility jurisdictions, the largest generating unit located in a load dominated area is normally considered as the most important generation resource of that area. Therefore, the largest generating unit in a load dominated area is frequently defined as RMR unit in the operating order to secure the operation of power system. In this study system, the largest generating unit on the island, Plant $\mathrm{F}$ having a 257.5 MW unit shown in Fig. 2, is used to provide the RMR service for system operation in order to secure the local island load under $N-1$ transmission contingencies. This largest unit (Plant F) is a gas-fired power plant with an expensive operating cost when the plant is required for RMR service under constraints of bulk power transmission system. As the island load continues to grow, the system becomes less secure as the $P_{\mathrm{M}}$ increases. As a result, potential operating cost in a form of RMR requirement will increase along with the load growth in order to secure the island system. The RMR generation cost to mitigate the system insecurity considered in this study was adapted from [26], which is derived from using a specified operating cost multiplied by the marginal probability $\left(P_{\mathrm{M}}\right)$.

The total RMR cost for Plant F consists of two cost components; fixed cost and variable cost. The fixed cost is associated with operating the plant (operation manning), which is assumed to be $200 \$ / \mathrm{h}$ representing the operation cost. The variable cost is associated with the fuel (gas) used in generating electricity. This cost varies with the generation output. For simplicity, an average gas price is assumed to be $72 \$ / \mathrm{MWh}$ representing the unit cost of energy production. A minimum generation output of Plant $\mathrm{F}$ to meet RMR requirements is set at $100 \mathrm{MW}$, which is the minimum generation required to cope with the $N-1$ security criterion, i.e. when the system encounters the marginatate $\left(P_{\mathrm{M}}\right)$. Therefore, the operating cost of Plant $\mathrm{F}$ obligated to the $\mathrm{RMR}$ service is equal to $\$ 72 \times 100 \mathrm{MW}+\$ 200=7400 \$ / \mathrm{h}$. By adopting the expected insecurity cost in [26], the RMR generation cost can be calculated by using $\$ 7400 \times P_{\mathrm{M}} \times 8760$.

\subsubsection{Customer damage cost reduction}

The energy loss and RMR costs described previously are the costs that directly incur to power utilities and are considered as the hard benefits. In contrast, customer damage cost due to power supply interruption is essentially impacted to end-users' side and is considered as the soft benefit to power utilities. For this reason, power utilities may or may not include it in the economic analysis of the project depending on different countries' regulations and jurisdictions.
Customer damage cost can be calculated by using the average unit interruption cost multiplied by the expected energy not supplied (EENS). The EENS is occurred when the system encounters the at-risk state, and therefore the EENS can represent the system risk deriving from a product of probability (i.e. unavailability) multiplied by its consequence (i.e. magnitude of load lost). The customer damage cost can be calculated by a product of EENS and the average unit interruption cost. It is assumed that the average unit interruption cost for the island load is equal to $10 \$ / \mathrm{kWh}$.

The energy loss, RMR generation cost, and customer damage cost of the two planning alternatives for the 15-year long range plan were calculated, and are summarized in Table 4. The benefits from advancing the transmission system reinforcement project are associated with the reductions in energy loss, in RMR cost, and in damage cost, which can be realized by directly comparing the all the three costs between both planning alternatives in the first four years. For example, the reduction in the energy loss in Year 1 is approximately 10 million dollars (i.e. 39.11-29.37) whereas the reduction in the RMR generation cost in Year 1 is approximately 0.5 million dollars (i.e. 0.54-0.06). It is obvious that the energy loss saving is a dominant benefit in this project. The values between the two alternatives shown in Table 4 are different only in the first four years as highlighted by the italic font in Alternative 2 when the transmission system reinforcement project has been advanced. The values from Years 5 to 15 are identical for both planning alternatives because the system configuration and conditions from Years 5 to 15 are exactly the same. Therefore, the benefits gained in terms of the reductions in energy loss, RMR cost, and damage cost can be observed from Years 1 to 4 only, and these benefits will be further considered in the economic analysis.

It should also be emphasized that the overall system reliability, from both security and adequacy aspects, has been enhanced since the RMR cost (security-related) and the customer damage cost (adequacy-related) are reduced with the transmission reinforcement project advancement.

\subsection{Economic analysis}

In order to appreciate whether or not the advancement of the new $230 \mathrm{kV}$ transmission project is economically justified, the economic comparison in a form of PV calculation between both planning alternatives is required. The PV describes how much a future sum of money is worth today. Time value of money implies that a dollar today is worth more than a dollar tomorrow. This means that a deferral of an investment project to the future would be more beneficial from financial saving aspect than investing it today based on the time value of money. As opposed to the 
Table 4 Energy loss, RMR cost and damage cost of two alternatives for 15-year long range plan

\begin{tabular}{|c|c|c|c|c|c|c|c|}
\hline \multirow[t]{2}{*}{ Year } & \multirow[t]{2}{*}{ Peak load (MW) } & \multicolumn{3}{|l|}{ Alternative 1} & \multicolumn{3}{|l|}{ Alternative 2} \\
\hline & & Energy loss $(\$ M)$ & RMR cost $(\$ M)$ & Damage cost $(\$ M)$ & Energy loss $(\$ M)$ & RMR cost $(\$ M)$ & Damage cost $(\$ M)$ \\
\hline 1 & 2390 & 39.11 & 0.54 & 0.36 & 29.37 & 0.06 & 0.02 \\
\hline 2 & 2419 & 40.18 & 0.57 & 0.41 & 29.92 & 0.06 & 0.02 \\
\hline 3 & 2448 & 41.26 & 0.63 & 0.48 & 30.48 & 0.07 & 0.03 \\
\hline 4 & 2477 & 42.41 & 0.68 & 0.55 & 31.00 & 0.08 & 0.03 \\
\hline 5 & 2507 & 31.70 & 0.09 & 0.03 & 31.70 & 0.09 & 0.03 \\
\hline 6 & 2537 & 32.37 & 0.10 & 0.03 & 32.37 & 0.10 & 0.03 \\
\hline 7 & 2567 & 32.98 & 0.11 & 0.04 & 32.98 & 0.11 & 0.04 \\
\hline 8 & 2598 & 33.55 & 0.12 & 0.04 & 33.55 & 0.12 & 0.04 \\
\hline 9 & 2629 & 34.36 & 0.13 & 0.05 & 34.36 & 0.13 & 0.05 \\
\hline 10 & 2661 & 35.21 & 0.14 & 0.06 & 35.21 & 0.14 & 0.06 \\
\hline 11 & 2693 & 36.07 & 0.17 & 0.07 & 36.07 & 0.17 & 0.07 \\
\hline 12 & 2725 & 37.05 & 0.19 & 0.08 & 37.05 & 0.19 & 0.08 \\
\hline 13 & 2758 & 38.08 & 0.22 & 0.10 & 38.08 & 0.22 & 0.10 \\
\hline 14 & 2791 & 39.12 & 0.25 & 0.12 & 39.12 & 0.25 & 0.12 \\
\hline 15 & 2824 & 40.22 & 0.28 & 0.15 & 40.22 & 0.28 & 0.15 \\
\hline
\end{tabular}

project deferral, investing on the new $230 \mathrm{kV}$ transmission project in advance by 4 years (Alternative 2: advanced plan) would incur higher monetary value than investing based on the normal schedule (Alternative 1: traditional plan). Therefore, this subsection investigates the present value of the two planning alternatives to determine whether it is worth advancing the investment project by 4 years or not. The following assumptions are used in the economic analysis:

1) Capital investment cost of the new $230 \mathrm{kV}$ transmission addition project is $\$ 180 \mathrm{M}$, which consists of constructing $17 \mathrm{~km}$ overhead line and $38 \mathrm{~km}$ submarine cable, and adding a $230 \mathrm{kV} 600$ MVA phase shifting transformer in series with the new transmission circuit.

2) Operating, maintenance and administration (OMA) cost is assumed to be a fixed percentage of the project investment cost, and the OMA cost rates increase with the year of in service (i.e. Years 1 to 10 at $0.25 \%$, Years 11 to 15 at $0.35 \%$, etc.)

3) In economic analysis, the hard benefits (energy loss and RMR cost reductions) as well as the soft benefit (customer damage cost reduction) are all considered.

4) Present value is calculated based on the value of money of Year 1 (i.e. when the island peak load exceeds $2371 \mathrm{MW}$ ).

5) Discount rate is $5 \%$.

The present value over the planning horizon is calculated by (1) as shown below [27]:
$P V=\sum_{j=1}^{m} \frac{A_{j}}{(1+r)^{j-1}}$

where $P V$ is the present value; $A_{j}$ is the expenditure in Year $j ; r$ is the discount rate; $m$ is the number of years considered in the planning horizon.

The PV calculations for the traditional plan (Alternative 1) and the advanced plan (Alternative 2) are presented in Tables 5 and 6, respectively. The economic analysis results indicate that the PV of the traditional plan yields $\$ 560.3 \mathrm{M}$, whereas the PV of the advanced plan is $\$ 551.5 \mathrm{M}$. The PV of the advanced plan is less expensive than the PV of the traditional plan indicating that the advancement of the project by 4 years is more beneficial. Therefore, it can be concluded that the advancement of the new $230 \mathrm{kV}$ transmission project by 4 years is economically justified. The main benefit of the project advancement, in this case, is due to the energy loss saving (reduction) when constructing the project 4 years earlier than the traditional plan. It is worth noting that both soft and hard benefits are included in Tables 5 and 6 , yielding the overall cost that addresses benefits from both power utility and customers' sides. A sensitivity study of advancing the project by 1 and 2 years from the traditional plan was also investigated, but the detailed analysis of this sensitivity study is not shown in order to avoid a lengthy paper. The PV of the advanced plan by 1 and 2 years earlier, respectively, are $\$ 557.3 \mathrm{M}$ and $\$ 555.8 \mathrm{M}$. These values indicate several million dollars can still be saved when compared to the traditional plan (\$ $560.3 \mathrm{M})$. 
Table 5 PV analysis of Alternative 1 for 15-year planning horizon

\begin{tabular}{|c|c|c|c|c|c|c|c|c|c|c|c|c|c|c|c|}
\hline \multirow[t]{2}{*}{ Parameters } & \multicolumn{15}{|l|}{ Year } \\
\hline & 1 & 2 & 3 & 4 & 5 & 6 & 7 & 8 & 9 & 10 & 11 & 12 & 13 & 14 & 15 \\
\hline 1. Capital cost $(\$ M)$ & - & - & - & - & 180.0 & - & - & - & - & - & - & - & - & - & - \\
\hline 2. OMA cost $(\$ M)$ & - & - & - & - & 0.5 & 0.5 & 0.5 & 0.5 & 0.5 & 0.5 & 0.5 & 0.5 & 0.5 & 0.5 & 0.6 \\
\hline 3. $\mathrm{RMR}$ cost $(\$ \mathrm{M})$ & 0.5 & 0.6 & 0.6 & 0.7 & 0.1 & 0.1 & 0.1 & 0.1 & 0.1 & 0.1 & 0.2 & 0.2 & 0.2 & 0.2 & 0.3 \\
\hline 4. Energy loss cost $(\$ M)$ & 39.1 & 40.2 & 41.3 & 42.4 & 31.7 & 32.4 & 33.0 & 33.5 & 34.4 & 35.2 & 36.1 & 37.1 & 38.1 & 39.1 & 40.2 \\
\hline 5. Damage cost $(\$ M)$ & 0.4 & 0.4 & 0.5 & 0.6 & 0.0 & 0.0 & 0.0 & 0.0 & 0.1 & 0.1 & 0.1 & 0.1 & 0.1 & 0.1 & 0.2 \\
\hline Total cost $(1+2+3+4+5)(\$ M)$ & 40.0 & 41.2 & 42.4 & 43.6 & 212.3 & 33.0 & 33.6 & 34.2 & 35.0 & 35.9 & 36.8 & 37.8 & 38.9 & 39.9 & 41.3 \\
\hline Total cost at discount rate $(\$ M)$ & 40.0 & 39.2 & 38.4 & 37.7 & 174.6 & 25.8 & 25.1 & 24.3 & 23.7 & 23.1 & 22.6 & 22.1 & 21.6 & 21.2 & 20.8 \\
\hline
\end{tabular}

Note: Damage costs in Years 5 to 8 are not exactly zero. The values shown as 0.0 due to being rounded to one digit after decimal point. PV is the sum of total cost at discount rate over 15 years

Table 6 PV analysis of Alternative 2 for 15-year planning horizon

\begin{tabular}{|c|c|c|c|c|c|c|c|c|c|c|c|c|c|c|c|}
\hline \multirow[t]{2}{*}{ Parameters } & \multicolumn{15}{|l|}{ Year } \\
\hline & 1 & 2 & 3 & 4 & 5 & 6 & 7 & 8 & 9 & 10 & 11 & 12 & 13 & 14 & 15 \\
\hline 1. Capital cost $(\$ M)$ & 180.0 & - & - & - & - & - & - & - & - & - & - & - & - & - & - \\
\hline 2. OMA cost $(\$ M)$ & 0.5 & 0.5 & 0.5 & 0.5 & 0.5 & 0.5 & 0.5 & 0.5 & 0.5 & 0.5 & 0.6 & 0.6 & 0.6 & 0.6 & 0.6 \\
\hline 3. RMR cost $(\$ M)$ & 0.1 & 0.1 & 0.1 & 0.1 & 0.1 & 0.1 & 0.1 & 0.1 & 0.1 & 0.1 & 0.2 & 0.2 & 0.2 & 0.2 & 0.3 \\
\hline 4. Energy loss cost $(\$ M)$ & 29.4 & 29.9 & 30.5 & 31.0 & 31.7 & 32.4 & 33.0 & 33.5 & 34.4 & 35.2 & 36.1 & 37.1 & 38.1 & 39.1 & 40.2 \\
\hline 5. Damage cost $(\$ M)$ & 0.0 & 0.0 & 0.0 & 0.0 & 0.0 & 0.0 & 0.0 & 0.0 & 0.1 & 0.1 & 0.1 & 0.1 & 0.1 & 0.1 & 0.2 \\
\hline Total cost $(1+2+3+4+5)(\$ M)$ & 209.9 & 30.5 & 31.0 & 31.6 & 32.3 & 33.0 & 33.6 & 34.2 & 35.0 & 35.9 & 36.9 & 38.0 & 39.0 & 40.1 & 41.3 \\
\hline Total cost at discount rate $(\$ M)$ & 209.9 & 29.0 & 28.1 & 27.3 & 26.6 & 25.8 & 25.1 & 24.3 & 23.7 & 23.1 & 22.7 & 22.2 & 21.7 & 21.3 & 20.8 \\
\hline
\end{tabular}

Note: Damage costs in Years 1 to 8 are not exactly zero. The values shown as 0.0 due to being rounded to one digit after decimal point. PV is the sum of total cost at discount rate over 15 years

\section{Conclusion}

A comprehensive approach to enhance the deterministic-based power grid planning utilizing system well-being analysis concept is illustrated in this paper. The methodology for translating the accepted deterministic criteria into the benchmark well-being indices is described. The combined deterministic and probabilistic framework, then, is used to determine an appropriate amount of the transmission capacity reserve requirement to cope with both generation and transmission uncertainties arisen within the system.

The transmission system planning application was demonstrated using a practical island system considering both technical and economic aspects. The planning outcomes obtained from the proposed technique indicate that the advancement of the system reinforcement project to accommodate the transmission capacity reserve can be economically justified. The project advancement can enhance the overall system reliability from both security and adequacy standpoints. The proposed technique can prove useful for system planners and decision makers in quantifying degrees of system security and adequacy improvements associated with the reinforcement project and then monetizing the overall system benefits inherited from the project for the economic analysis purposes.

Acknowledgements This research work is financially supported by TGGS Research Fund (No. TGGS-NEW-58-01).

Open Access This article is distributed under the terms of the Creative Commons Attribution 4.0 International License (http:// creativecommons.org/licenses/by/4.0/), which permits unrestricted use, distribution, and reproduction in any medium, provided you give appropriate credit to the original author(s) and the source, provide a link to the Creative Commons license, and indicate if changes were made.

\section{Appendix A}

Table A1 presents the forced outage rates of all generating units and bulk transmission circuits supplying the island system as presented in Fig. 2. 
Table A1 Individual component FORs

\begin{tabular}{lll}
\hline Component & Capacity (MW) & FOR \\
\hline Generating unit in Plant A & 27 & 0.0150 \\
Generating units in Plant B & 20 & 0.0150 \\
Generating unit in Plant C & 18 & 0.0250 \\
Generating units in Plant D & 23.5 & 0.0250 \\
Generating units in Plant E & 30 & 0.0150 \\
Generating unit in Plant F & 257.5 & 0.0750 \\
Generating unit in Plant G & 162.5 & 0.0550 \\
$500 \mathrm{kV}$ circuit No. 1 & 1200 & 0.0041 \\
$500 \mathrm{kV}$ circuit No. 2 & 1200 & 0.0005 \\
$230 \mathrm{kV}$ circuit & 600 & 0.0259 \\
\hline
\end{tabular}

\section{References}

[1] Billinton R, Bao H, Karki R (2008) A joint deterministicprobabilistic approach to bulk system reliability assessment. In: Proceedings of the 10th international conference on probabilistic methods applied to power systems, Rincon, Puerto Rico, 25-29 May 2008, 8 pp

[2] Reppen ND (2004) Increasing utilization of the transmission grid requires new reliability criteria and comprehensive reliability assessment. In: Proceedings of the 8th international conference on probabilistic methods applied to power systems, Iowa, USA, 19-22 September 2004, 6 pp

[3] Billinton R, Mo R (2004) Deterministic/probabilistic contingency evaluation in composite generation and transmission systems. In: Proceedings of the 2004 IEEE power engineering society general meeting, Denver, USA, 6-10 June 2004, 6 pp

[4] Billinton R, Lian G (1994) Composite power system health analysis using a security constrained adequacy evaluation procedure. IEEE Trans Power Syst 9(2):936-941

[5] Allan RN, Billinton R (2000) Probabilistic assessment of power systems. Proc IEEE 88(2):140-162

[6] Melo ACG et al (2006) An attempt to construct a bridge between deterministic and probabilistic approaches in transmission expansion planning - the PLANTAC model. CIGRE, Paris, France, 27 August-1 September 2006, 8 pp

[7] Li WY, Choudhury P (2007) Probabilistic transmission planning. IEEE Power Energy Mag 5(5):46-53

[8] Li WY (2015) Framework of probabilistic power system planning. CSEE J Power Energy Syst 1(1):1-8

[9] EPRI (2001) Assessment Methods and Operating Tools for Grid Reliability, Final Report No. 1001408

[10] EPRI (2003) Moving Toward Probabilistic Reliability Assessment Methods, Final Report No. 1002639

[11] Pei Z, Liang M, Hopkins L et al (2007) Utility experience performing probabilistic risk assessment for operational planning. In: Proceedings of the 14th international conference on intelligent system applications to power systems, Taiwan, China, 4-8 November 2007, 6 pp

[12] Wangdee W, Li WY, Billinton R (2015) Locational transmission capacity reserve determination using system well-being analysis. Electr Power Syst Res 119:329-336

[13] Productivity Commission (2013) Current transmission reliability and planning framework. http://www.pc.gov.au/inquiries/ completed/electricity/report/31-electricity-appendixf.pdf.

Access 19 Dec 2016
[14] Golmohamadi H, Ramezani M, Bashian A et al (2014) Riskbased maintenance scheduling of generating units in the deregulated environment considering transmission network congestion. J Mod Power Syst Clean Energy 2(2):150-162

[15] Billinton R, Allan RN (1996) Reliability evaluation of power systems, 2nd edn. Plenum, New York

[16] Goel L, Feng C (1999) Well-being framework for composite generation and transmission system reliability evaluation. IEE Proc-Gener Transm Distrib 146(5):528-534

[17] Leite SAM, Resende LC, Manso LAF et al (2004) Well-being analysis for composite generation and transmission systems. IEEE Trans Power Syst 19(4):1763-1770

[18] Wangdee W, Billinton R (2006) Bulk electric system well-being analysis using sequential Monte Carlo simulation. IEEE Trans Power Syst 21(1):188-193

[19] Fink LH, Carlsen K (1978) Operating under stress and strain. IEEE Spectr 15(3):48-53

[20] EPRI (1987) Composite-system reliability evaluation: phase 1-scoping study. Technical Report: EPRI EL-5290

[21] Billinton R, Khan E (1992) A security based approach to composite power system reliability evaluation. IEEE Trans Power Syst 7(1):65-72

[22] Billinton R, Li WY (1994) Reliability assessment of electrical power systems using Monte Carlo methods. Plenum Publishing, New York

[23] Wangdee W, Li WY, Shum W et al (2009) Assessing transfer capability requirement for wind power generation using a combined deterministic and probabilistic approach. In: Proceedings of the 2009 IEEE power engineering society general meeting, Calgary, Canada, 26-30 July 2009, 8 pp

[24] BC Hydro Current Practice (2013) Brief on determining transmission capacity for intermittent resources. http://ewh.ieee.org/ cmte/pes/rrpa/RRPA_files/LBP20130711/20130725\% 20LOLEWG\%20Item-02e\%20BC-Hydro\%20Brief_Criteria_ LOLE_ELCC.pdf. Accessed 19 Dec 2016

[25] BC Hydro (2014) Operating order 7T-12: reliability must run generation requirements. https://www.bchydro.com/content/ dam/BCHydro/customer-portal/documents/corporate/suppliers/ transmission-system/system_operating_orders/7T12.pdf. Accessed 19 Dec 2016

[26] Billinton R, Wangdee W (2006) A combined bulk electric system reliability framework using adequacy and static security indices. J Electr Eng Technol 1(4):414-422

[27] Li WY (2014) Risk assessment of power systems: models, methods, and applications, 2nd edn. Wiley, Hoboken

Wijarn WANGDEE received the B.Eng. degree from Chulalongkorn University, Thailand, in 1999 and the M.Sc. and Ph.D. degrees from the University of Saskatchewan, Canada, in 2002 and 2005, respectively. From 2006 to 2013, he worked as a system planning engineer for BC Hydro and Power Authority, Vancouver, Canada. He is currently an assistant professor at the Sirindhorn International ThaiGerman Graduate School of Engineering (TGGS), King Mongkut's University of Technology North Bangkok (KMUTNB), Thailand. His research interests include power system reliability, power system dynamics, renewable energy integration and synchrophasor applications. 